\title{
Design of Data Acquisition and Transmission System based on Single Chip Microcomputer
}

\author{
1, aWANG Xiaohua \\ ${ }^{1}$ Institute of Applied Electronics, Chongqing College of Electronic Engineering, Chongqing, \\ 401331 \\ a12934236@qq.com
}

\begin{abstract}
Keywords: Data Acquisition, Transmission System, Single Chip Microcomputer
\end{abstract}
\begin{abstract}
Data acquisition technology is a practical electronic technology, which is widely used in signal detection, signal processing, instrumentation and other fields. In recent years, with the continuous development of digital technology, data acquisition technology also presents a characteristic of faster processing and more channels. The data acquisition is to measure and control the physical quantity such as temperature, pressure, flow, speed, displacement, light intensity, sound, etc., Convert the physical quantity into electric signal of simulation physical quantity through the sensor, then process the analog electric signal and convert it into signal that computer can recognize the digital, then sent to the computer processing, storage, transmission and display. This paper describes the hardware and software system construction of data acquisition and transmission system.
\end{abstract}

\section{Introduction}

Data collection technology began to appear in the 1950s. The data acquisition system used in military affairs in 1956 was successfully developed in the United States. This system has two advantages in use and on the one hand, it can not use the relevant detection in the application process File, on the other hand without the professional training of personnel can also operate on it. Because the system has a certain high speed and flexibility, can complete many other methods can not complete the experimental task, so the US military has been recognized. Beginning in the late 1970s, well-developed data acquisition devices were already available in foreign countries. In the late 1970s, with the development of micro-computer technology, a new type of data acquisition equipment, which is a collection of devices, digital instruments and micro-computer combination, because its function than the traditional data acquisition system strong, so quickly get the application and promotion. But this time the data acquisition system began to fall into two categories, one is designed for laboratory use, the other mainly used in industrial production. By the 1980s, computer technology has developed rapidly, and data acquisition systems have begun to move away from the original special features, and gradually to the versatility. This era of data acquisition system has begun to develop into a modular structure, the use of the process can increase or decrease the number of terminals in the data communication has been a field bus, computer interface technology and other new technologies, and because of the use of The modular design makes the whole system combination, the use of more convenient and simple. From the 20th century, late 80's, data acquisition system, the composition of the structure of a great change in the design of software and hardware combined with the idea began to appear in the system design process, at this stage data acquisition system hardware generally by the industrial computer, And large-scale integrated circuit composition, the software is mainly responsible for the coordination and management of the entire system. This design not only saves production costs, reduce the volume, but also effectively improve the system efficiency. Since the 1990s, data collection has become a professional technology, the emergence of a high-precision, high reliability, high-speed single-chip data acquisition system (DAS), this system can achieve accuracy of 16-bit collection, acquisition Speed can reach hundreds of thousands of times per second, it has been widely used in military equipment, aerospace, industrial field and other fields. 


\section{Overall System Design}

By comparing the advantages and disadvantages of similar systems at home and abroad, and detailed analysis of the system, in view of the technical requirements of the system, this paper uses the STC89C52 microcontroller as the core, designed and issued a set of data collection, wireless data transmission, data monitoring display of the multi-functional group-hui parameter monitoring system, the system should have universal applicability. The following general design scheme is proposed:

1) To STC89C52 microcontroller as the core, combined ADC0809 analog-to-digital conversion chip and E2PR0M AT24C08 chip set up data acquisition and storage systems.

2) Using LCD1602 LCD display real-time display data acquisition system design, the use of MAX232 complete TTL level and RS232 level conversion.

3) The use of Shenzhen Technology Co., Ltd. JZ877 low-power wireless transmission module to build wireless data transmission network.

4) The use of VBMSCOMM control host data display monitoring window, real-time data monitoring.

5) Software programming using C language: modular programming, high efficiency, clear structure and logic, good readability, good portability.

\section{System Hardware Design}

SCM system consists of the smallest work: First, STC89C52 microcontroller, power supply, the second is the clock circuit, reset circuit and interface. STC89C52 single-chip power supply is for the DC 5V. In the microcontroller system, generally in the XTAL1 and XTAL2 pin of a crystal and two capacitors, thus forming a chip shock mode, chip shock is because the 51 core microcontroller which has a high gains inverting amplifier, an external one After the crystal oscillator, they form the self-excited oscillator and generate the shock pulse. Its structure is not shown in Figure 3-3. In the choice of the general choice of crystal quartz crystal, the size of 1.2 - 12MHZ between the size of the capacitor as long as the $5-30 \mathrm{pF}$ can be between the size of the capacitor can play the role of fine-tuning frequency, but the size of the capacitor and the crystal load impedance Between the coincidence, otherwise the clock circuit will be difficult to start.

SCM system, generally have a reset circuit, so that the error in the microcontroller code by the user to reset their own, for the 51-core microcontroller is a high level reset is valid. In the ordinary one-chip computer system, a resistor, a capacitor, a manual button constitutes a microcontroller reset system.

In the smallest single-chip microcomputer system, the general I/O resources into a scalable interface, the use of these interface resources and the microcontroller can be connected with a number of external devices or devices, such as: A/D, LCD, LED, etc. In addition, you can use these interface resources to extend the data memory or program memory and other off-chip storage devices.

Acquisition circuit board in the work of a stable power supply is essential, so the schematic design must ensure that the power supply circuit design is reasonable. This acquisition system used in the DC power supply schematic diagram, in this figure $220 \mathrm{~V}$ of the grid voltage through the step-down transformer down to $18 \mathrm{~V}$ AC, and then through the rectifier bridge to make AC into DC, and then filtered through the capacitor and the three-terminal regulator chip And so the final output stable and reliable DC voltage.

Because the system uses ADC0809 analog signal input range is 0-5V, and in the splicing process, the firing current and voltage signals are not in this range, so do not get from the device current and voltage signals directly to the input ADC0809, the need for feedback from the dry device on the current and voltage signals to the necessary signal conditioning to meet the A/D conversion chip parameter conversion range, and then through the MCU control of the A/D conversion chip and then adjust the parameters Signal for A/D conversion. 


\section{System Software Design}

In the SCM software development is often used in computer language assembly language and C language, the use of assembly language for software development can take full advantage of all the hardware features of the microcontroller and can directly control the hardware. It has the advantages of high efficiency in the process of writing programs and precise control of computer hardware, but it can not be structured programming, the preparation of the program portability and poor readability, while debugging process is also more complex. C language as a high-level computer language, use it for programming simple syntax, easy operation and flexible, but also for structured programming, can generate compact code, the target code generation efficiency is only $10 \%-20 \%$ lower than the assembly language. In addition C language program and assembly language program compared with more readable, portability, but also simple and convenient. Therefore, the software development of the project uses $\mathrm{C}$ language programming, program compilation and object code generation tool for the German KEIL Software company KEIL C51 software.

In order to make the system program structure clear, easy to write debugging, in the software development process uses modular programming method. Data acquisition system software program includes the following main modules: the main program, the system initialization program, ADC0809 parameter acquisition subroutine, LCD 1602 parameter display subroutine, AT24C08 parameter storage subroutine and RS232 serial communication subroutine. Among them, the system initialization includes STC89C52 microcontroller pin assignment settings, global variable declaration and initialization, each subroutine module parameter declaration and initialization, etc., and the main program is the core of all system software, the main completion of each subroutine call, control the whole data acquisition system is running normally.

The system main program of the implementation of the process is as follows: First, Hui machine start, acquisition system power-on reset, the system initialization, then the system through the detection of dry current, voltage to determine the state machine work, the process is collected by the system The transfer of current, voltage and program in advance to set the current and voltage to complete the comparison, when the judge has been in the normal operation of the state machine, the main program will start A/D conversion subroutine A/D sampling, and then Storage, display and transmission of parameters. When the burning current for a period of time to zero after the end of the main program, into the acquisition, to determine whether the transfer began, after the start of the swing into the cycle.

The main task of this thesis is to design a set of data acquisition and wireless data transmission system, the data acquisition system is the focus of this paper, and digital conversion is the core of data acquisition system. In this design, the use of ADC0809 as the parameters of the analog conversion chip, ADC0809 internal structure shown in Figure 4-2, its related characteristics and pin configuration parameters please refer to the relevant content of the second chapter of this paper. Figure 4-2 can be seen that the A/D total of eight analog input channels, the program set by A, B, C three-channel address selection to determine IN1 and IN2, respectively, voltage, current collection channel, through the software settings Two channels are automatically cycled and sampled. First, the IN1 channel is selected to glow the voltage signal sampling, and open the interrupt service routine, the interrupt service routine using the timer work after the delay Is read three-state output buffer voltage conversion results, and change the channel address, Next, the IN2 channel is turned on to start sampling the current signal and turn on the interrupt service routine. It also reads the current conversion result in the three-state output buffer and changes the channel address after the timer operation delay IS. So the sampling cycle can be achieved on the current and voltage parameters of the stop collection.

AT24C08 is a I2C bus interface with the E2PR0M chip, but STC89C52 microcontroller and 51 microcontroller has an internal architecture similar to this microcontroller does not have I2C bus interface, so when the microcontroller in the STC89C52 control system to use AT24C08 only through software simulation I2C bus timing, and this simulation must strictly follow the I2C bus timing diagram in the use of AT24C08 for data storage and reading, just call the various functions 
can be completed. The following is the timing diagram and the program for the relevant I2C bus.

According to the design requirements of the experiment, after the completion of data collection and wireless transmission work on the host to the acquisition of the transmission parameters of the display, analysis and processing, the test used to prepare the host monitoring interface program VB, the design of the host monitoring interface. The whole interface contains the current and voltage value display and related statistical window. The display interface mainly uses the serial communication function of MSCOMM control in VB to realize the communication between host PC and wireless data transmission module. In order to test the transmission parameter monitoring system designed in this paper, the system debugging is carried out at the end of the paper. The debugging includes the following contents: First, JZ877 low power wireless data transmission module debugging; Second, the host display interface Function debugging; Third, the overall system debugging. Through system debugging, we can find out the shortcomings of the system and provide suggestions for the follow-up system improvement. Debugging process and the following data obtained by the analysis and discussion.

\section{Conclusion}

Data acquisition technology and wireless transmission system is an important branch of information science and it has important applications in many ways. The data acquisition and wireless transmission system based on single-chip microcomputer adopts the module of high-performance wireless transceiver module, AD conversion, single-chip microcomputer and serial communication to realize a reliable and high-efficient data acquisition and transmission system. The circuit is simple and has stable performance, high accuracy and sensitivity, low cost. So it is suitable for practical applications.

\section{References}

[1] Li Guozhu. Design of Data Acquisition System based on Single Chip Microcomputer and USB Interface [J]. Modern Electronics, 2014 (04)

[2] Zhang Xuedong. Applications of AT89C51 single chip on wireless data transmission [J]. Heilongjiang Science and Technology Information, 2011 (07)

[3] Wei Qingtao. Wireless data transmission system based on single-chip micro-computer [J] .Computer and Modernization, 2007 (04)

[4] Zhu Fang. Data acquisition system design Based on SCM [J]. Journal of Chongqing University of Science and Technology (Natural Science Edition), 2012 (02)

[5] Jiang Nianping, Huang Wenjie. Data Acquisition System and Intelligent Management based on Wireless Transmission [J]. Micro-computer Information, 2009 (22) 